\title{
Perspective Piece \\ Next-Generation Sequencing Analysis of Pathogenic Leptospira: A Way Forward for Understanding Infectious Disease Dynamics in Low/Middle-Income, Disease-Endemic Settings
}

\author{
Suneth B. Agampodi ${ }^{1,2 \star}$ and Joseph M. Vinetz ${ }^{1}$ \\ ${ }^{1}$ Section of Infectious Diseases, Department of Internal Medicine, Yale University School of Medicine, New Haven, Connecticut; ${ }^{2}$ Department of \\ Community Medicine, Faculty of Medicine and Allied Sciences, Rajarata University of Sri Lanka, Saliyapura, Sri Lanka
}

\begin{abstract}
In the current genomic era, knowledge of diversity of Leptospira, the spirochetal agents of leptospirosis, is changing rapidly. Next-generation sequencing has decreased in price and increased in scale, with the potential to democratize large-scale analysis of pathogens in resource-limited, low/middle-income (LMIC) regions. Consequently, the molecular classification of Leptospira, a pathogen disproportionately affecting LMIC countries, has changed dramatically over the last decade. Leptospira classification and molecular understandings of pathogen diversity have rapidly evolved, now most precisely based on core genome analysis supplemented by new insights provided by cultureindependent methods directly using body fluids such as blood and urine. In places where leptospirosis disease burden is highest, genomic technologies have not been available, and serology-based methods remain the mainstay of leptospiral classification. Understanding the epidemiology, pathogenesis, and ultimately new approaches to treating and preventing leptospirosis requires detailed knowledge of regionally circulating Leptospira in highly endemic settings. Next-generation sequencing-based, culture-independent typing overcomes the limitation of culture isolation of Leptospira from clinical samples, with promise of providing public health-actionable information applicable to leptospirosis-endemic LMIC settings.
\end{abstract}

Knowledge of pathogen diversity is an important component of infectious disease control programs. Leptospirosis, a globally widespread zoonotic disease, is caused by the most diverse bacteria genera. The genus Leptospira, of the phylum Spirochaetales, comprised a large number of infectious and noninfectious (saprophytic) species. ${ }^{1,2}$ Next-generation sequencing (NGS) has the potential to rapidly obtain detailed leptospiral genomic data in a high throughput and scalable way, for example, enabling whole genome sequencing-based concatenated core genome-based generation of phylogenic trees and multi-locus sequence typing (cgMLST). Such analyses have already led to a significant change in the taxonomy of Leptospira, for example, recently adding 43 new species to the previously known 24 species. ${ }^{1,3-5}$

The serological classification of Leptospira was first proposed in $1954,{ }^{6}$ which depends on the structural heterogeneity of the carbohydrate component (O-antigen) of Leptospira lipopolysaccharide. ${ }^{7}$ The proposed classification has evolved to include more than 300 serovars in 32 serogroups. ${ }^{8}$ Serological analysis of serovars and serogroups (comprising antigenically related serovars), which does not have species-level taxonomic status, has traditionally been used to classify leptospiral isolates. The labor-intensive crossagglutination absorption test using the microscopic agglutination test (MAT) with live Leptospira and corresponding rabbit antisera has traditionally been the method of choice for assigning serovars, but has become difficult to obtain from reference laboratories. ${ }^{9}$

The MAT is also used for the diagnosis of leptospirosis. It depends on serovar/serogroup-dependent reactions, with sera ideally obtained from acute illness and convalescence.

*Address correspondence to Suneth B. Agampodi, Section of Infectious Diseases, Department of Internal Medicine, Yale University School of Medicine, Yale University, Winchester Bldg., 25 York St., Rm. 428, New Haven, CT 06510. E-mails: suneth.agampodi@yale.edu or sunethagampodi@yahoo.com
Either a 4-fold rise in titer or a single high titer with an empirically determined cutoff based on the level of regional endemicity may be considered diagnostic in an appropriate clinical context. Microscopic agglutination test diagnostic panels comprise live Leptospira designed to represent known pathogenic serogroups and/or circulating serovars from a regionally optimized panel. In many countries with high incidence of leptospirosis, the panel of Leptospira is not optimized for the country because knowledge of circulating leptospires is not known. Leptospira isolation is fundamental to this process. Leptospira are fastidious and difficult to isolate and require specialized media and microscopy, and preclude culture and isolation in most endemic settings. Hence, the antigen panel used in the diagnostic process is often not regionally optimized. To improve diagnostic test sensitivity, a standard WHO/CDC MAT panel, comprising a larger number of represented serogroups, has recommended for settings where the leptospiral diversity is expected to be high but where knowledge of circulating serovars is incomplete or unknown.

The human leptospirosis literature commonly reports that the highest agglutination titer in the MAT indicates the infecting serovar; this is problematic. ${ }^{10}$ Many case reports (only a few examples cited here) $)^{11-13}$ and large hospital- ${ }^{14}$ and community-based studies ${ }^{15}$ have referred to the MAT titer to infer the infecting serovar. In 2003, using 151 culture-positive human leptospirosis cases, Levett demonstrated that the sensitivity of serogroup-level identification was less than $50 \% .{ }^{10}$ Another study carried out in Thailand using 101 culture-positive patients showed a $33 \%$ specificity of the infecting serovar identification. ${ }^{16}$ Because the standard WHO/ CDC MAT panel is the preferred choice in many endemic countries, the highest reacting strain in the panel may represent only the serogroup, not the infecting serovar. As Levett's work shows, even at the serogroup level, inferences of the infecting serovar from the MAT of patient sera are inaccurate. The cross-reactivity between different serogroups is high when the titer level is high. The comparison of MAT proficiency 
testing from 37 laboratories in 23 countries in 2002 also confirmed that the serogroup identification could be problematic. ${ }^{17}$ During the proficiency test, samples from four known serogroups were sent to laboratories, and only $57 \%$ of the laboratories had identified all four serogroups correctly partly because their MAT panels did not contain a serovar within the serogroup concerned. ${ }^{17}$ Since these observations, this issue has been discussed widely, and in general, researchers generally agree that the MAT should not be used to infer the infecting serovar. Delineating infecting serovar remains important for understanding and tracing infection source for disease control.

Although molecular methods are widely available for diagnosis, genus-level PCR does not differentiate pathogenic serovars; serovar-specific PCR remains elusive. Thus, it is important to develop efficient methods to precisely identify leptospiral serovars, thus replacing imprecise serology. Dissemination of robust molecular/genomic methods and protocols to precisely identify infecting Leptospira at the species and serovar levels clearly has the potential to be an important adjunct to culture isolation. Accuracy of identifying infecting Leptospira has clinical, public health, epidemiological, and agricultural implications for treatment, control, and prevention. Culture-independent classification at the species level was attempted with mixed results in several studies. ${ }^{18-22}$ The usual molecular targets used for this purpose includes $16 \mathrm{~s}$ ribosomal RNA gene, SecY, and flaB genes. Subspecies-level identification of infecting Leptospira was attempted mostly using different multi-locus sequence typing schemes ${ }^{23-25}$ but remains inefficient.

Because basic knowledge on pathogen diversity is essential for disease control programs, culture isolation of Leptospira still should be attempted despite its inherent challenges in endemic places. Coordinated, adequately resourced programs in highly endemic countries are needed to address this public health gap. These isolates will have multiple uses including validation of culture-independent methods, including the use of NGS with high-resolution sequencing platforms to overcome the challenge of culture isolation. Classification methods such as cgMLST typing, genomic single nucleotide polymorphisms with high discriminatory power, and virulence gene content will result from this work. Recent reductions in the cost of NGS in combination with the development of efficient bioinformatic analysis pipelines have the potential to enable genomic-level Leptospira analysis for researchers in low/middle-income (LMIC) regions. ${ }^{26}$ Newer technologies such as those offered by Oxford Nanopore Technologies have reduced the capital costs for equipment enough that researchers in leptospirosis-endemic regions have to access molecular testing. Researchers need to be aware of the potential for NGS-related resources to advance genomic knowledge of Leptospira and etiological agents of other infectious diseases in the LMIC setting.

Received November 28, 2020. Accepted for publication January 24, 2021.

Published online March 22, 2021.

Financial support: This work was supported by U.S. Public Health Service, National Institute of Allergy and Infectious Diseases of the National Institutes of Health, through grant number U19Al115658.

Authors' addresses: Suneth B. Agampodi, Section of Infectious Diseases, Department of Internal Medicine, Yale University School of Medicine, New Haven, CT, and Department of Community Medicine,
Faculty of Medicine and Allied Sciences, Rajarata University of Sri Lanka, Saliyapura, Sri Lanka, E-mails: suneth.agampodi@yale.edu or sunethagampodi@yahoo.com. Joseph M. Vinetz, Section of Infectious Diseases, Department of Internal Medicine, Yale University School of Medicine, New Haven, CT, E-mail: joseph.vinetz@yale.edu.

\section{REFERENCES}

1. Vincent AT et al., 2019. Revisiting the taxonomy and evolution of pathogenicity of the genus Leptospira through the prism of genomics. PLoS Negl Trop Dis 13: e0007270.

2. Fouts DE et al., 2016. What makes a bacterial species pathogenic? Comparative genomic analysis of the genus Leptospira. PLoS Negl Trop Dis 10: e0004403.

3. Thibeaux R, Iraola G, Ferres I, Bierque E, Girault D, Soupe-Gilbert ME, Picardeau M, Goarant C, 2018. Deciphering the unexplored Leptospira diversity from soils uncovers genomic evolution to virulence. Microb Genom 4: e000144.

4. Guglielmini J, Bourhy $P$, Schiettekatte $O$, Zinini F, Brisse S, Picardeau M, 2019. Genus-wide Leptospira core genome multilocus sequence typing for strain taxonomy and global surveillance. PLoS Negl Trop Dis 13: e0007374.

5. Casanovas-Massana A et al., 2020. Leptospira yasudae sp. nov. and Leptospira stimsonii sp. nov., two new species of the pathogenic group isolated from environmental sources. Int $J$ Syst Evol Microbiol 70, 1450-1456.

6. Wolff JW, Broom JC, 1954. The genus Leptospira Noguchi, 1917; problems of classification and a suggested system based on antigenic analysis. Doc Med Geogr Trop 6: 78-95.

7. Kalambaheti T, Bulach DM, Rajakumar K, Adler B, 1999. Genetic organization of the lipopolysaccharide $\mathrm{O}$-antigen biosynthetic locus of Leptospira borgpetersenii serovar Hardjobovis. Microb Pathog 27: 105-117.

8. Levett PN, 2015. Systematics of leptospiraceae. Curr Top Microbiol Immunol 387: 11-20.

9. Martin L, Pettit A, 1918. Sero-diagnostic de la spirochaetose icterohaemorrhagique. Bull Mem Soc Med Hop Paris 42: 672-675.

10. Levett PN, 2003. Usefulness of serologic analysis as a predictor of the infecting serovar in patients with severe leptospirosis. Clin Infect Dis 36: 447-452.

11. Lecour H, Miranda M, Magro C, Rocha A, Goncalves V, 1989. Human leptospirosis-a review of 50 cases. Infection 17: 8-12.

12. Gollop JH, Katz AR, Rudoy RC, Sasaki DM, 1993. Rat-bite leptospirosis. West J Med 159: 76-77.

13. Torre D, Giola M, Martegani R, Zeroli C, Fiori GP, Ferrario G, Bonetta G, 1994. Aseptic meningitis caused by Leptospira australis. Eur J Clin Microbiol Infect Dis 13: 496-497.

14. Agampodi SB, Thevanesam V, Wimalarathna H, Senarathna $T$, Wijedasa $\mathrm{MH}, 2008$. A preliminary study on prevalent serovars of leptospirosis among patients admitted to teaching hospital, Kandy, Sri Lanka. Indian J Med Microbiol 26: 405-406.

15. Rajapakse $S$ et al., 2020. Seroprevalence of leptospirosis in an endemic mixed urban and semi-urban setting-A communitybased study in the district of Colombo, Sri Lanka. PLoS Negl Trop Dis 14: e0008309.

16. Smythe LD et al., 2009. The microscopic agglutination test (MAT) is an unreliable predictor of infecting Leptospira serovar in Thailand. Am J Trop Med Hyg 81: 695-697.

17. Chappel RJ, Goris M, Palmer MF, Hartskeerl RA, 2004. Impact of proficiency testing on results of the microscopic agglutination test for diagnosis of leptospirosis. J Clin Microbiol 42: 5484-5488.

18. Perez J, Goarant C, 2010. Rapid Leptospira identification by direct sequencing of the diagnostic PCR products in New Caledonia. BMC Microbiol 10: 325.

19. Chiani $Y$, Jacob P, Varni V, Landolt N, Schmeling MF, Pujato N, Caimi K, Vanasco B, 2016. Isolation and clinical sample typing of human leptospirosis cases in Argentina. Infect Genet Evol 37: 245-251.

20. Philip $N$ et al., 2020. Leptospira interrogans and Leptospira kirschneri are the dominant Leptospira species causing human leptospirosis in Central Malaysia. PLoS Negl Trop Dis 14: e0008197. 
21. Koizumi N, Gamage CD, Muto M, Kularatne SA, Budagoda BD, Rajapakse RP, Tamashiro H, Watanabe H, 2009. Serological and genetic analysis of leptospirosis in patients with acute febrile illness in Kandy, Sri Lanka. Jpn J Infect Dis 62: 474-475.

22. Agampodi SB et al., 2011. Leptospirosis outbreak in Sri Lanka in 2008: lessons for assessing the global burden of disease. Am J Trop Med Hyg 85: 471-478.

23. Agampodi SB, Moreno AC, Vinetz JM, Matthias MA, 2013. Utility and limitations of direct multi-locus sequence typing on qPCRpositive blood to determine infecting Leptospira strain. Am J Trop Med Hyg 88: 184-185.
24. Weiss S, Menezes A, Woods K, Chanthongthip A, Dittrich S, Opoku-Boateng A, Kimuli M, Chalker V, 2016. An extended multilocus sequence typing (MLST) scheme for rapid direct typing of Leptospira from clinical samples. PLoS Negl Trop Dis 10: e0004996.

25. Varni V, Chiani Y, Nagel A, Ruybal P, Vanasco NB, Caimi K, 2018. Simplified MLST scheme for direct typing of Leptospira human clinical samples. Pathog Glob Health 112: 203-209.

26. Schwarze K, Buchanan J, Taylor JC, Wordsworth S, 2018. Are whole-exome and whole-genome sequencing approaches cost-effective? A systematic review of the literature. Genet Med 20: 1122-1130. 